\title{
IMPACT OF STARK SHIFTS ON THE RADIATION COOLING OF CU-DOMINATED PLASMAS
}

\author{
Pierre Corfdir*, Gabriel Lantz, Markus Abplanalp, \\ Thierry Delachaux, Frank Kassubek, Patrick Sütterlin, \\ MATTHIAS BATOR
}

\author{
ABB Corporate Research, Segelhofstrasse 1K, 5405 Baden-Dättwil, Switzerland \\ * pierre.corfdir@ch.abb.com
}

\begin{abstract}
We study the impact of Stark line shifts reported recently for $\mathrm{Cu}$ I transitions on the radiative cooling of $\mathrm{Cu}$-dominated plasmas. The observed detuning in absorption between the hot core and cold shell of the arc leads to a reduction in radiation reabsorption compared to the case where Stark line shifts are neglected. Using a modeling based on a phenomenological treatment of the Stark line shift, we show that this reduction is below $2 \%$.
\end{abstract}

Keywords: $\mathrm{Cu}$ plasmas, Stark effect, radiation cooling, radiative transfer equation, spectroscopy.

\section{Introduction}

A proper modeling of current interruption in lowvoltage breakers requires an accurate treatment of the radiation of $\mathrm{Cu}$ vapors produced by the evaporation of the contacts of the devices [1]. In that purpose, knowledge of the oscillator strengths and Stark broadening parameters of the optical transitions of the atoms and ions constituting the plasma is necessary. While Stark line shifts are usually neglected when computing the absorption of $\mathrm{Cu}$ plasmas [2], we have recently reported that $\mathrm{Cu}$ I $3 d^{9} 4 s 5 s$ transitions exhibit a Stark shift of about $0.1 \mathrm{~nm}$ with increasing temperatures from 5000 to $10000 \mathrm{~K}$ [3].

In this paper, we investigate the impact of the reported Stark line shifts on the radiation cooling of $\mathrm{Cu}$-dominated electric arcs. While shifts of about $0.1 \mathrm{~nm}$ are observed with increasing plasma temperature from 5000 to $10000 \mathrm{~K}$, the wavelength of $\mathrm{Cu} \mathrm{I}$ $3 d^{9} 4 s 5 s$ remain essentially constant for higher temperatures. Using these experimental results, we compute the radiation intensity by a $\mathrm{Cu}$ arc with and without Stark line shift and show that these shifts have only a minor impact on the radiation cooling of the arc.

\section{Experimental Methods}

All experiments have been performed on arcs forming between a $5 \mathrm{~mm}$ diameter cylindrical and a ring (inner diameter of $20 \mathrm{~mm}$ ) Cu electrodes separated by $58 \mathrm{~mm}$. The interface of the cylindrical electrode with air is limited to $17 \mathrm{~mm}^{2}$ using a Macor cap. We measured the arc current and the voltage difference between the electrodes using closed loop Hall and differential probes, respectively. Using an interleaved Buck converter with three parallel lines, we could generate a current profile consisting of a linear increase from 0 to $2 \mathrm{kA}$ in $1 \mathrm{~ms}$, followed by a $19 \mathrm{~ms}$ long plateau with a constant current of $2 \mathrm{kA}$, and a linear decrease to zero over $30 \mathrm{~ms}$. Light emission from the arc discharge was detected with a high-speed camera with frame rate and exposure time of 6400 frames per second and $1 \mu \mathrm{s}$, respectively. For spectroscopy measurements, we collected the light emitted by a spot centered on the $z$-axis $3.5 \mathrm{~mm}$ away from the cathode surface and analyzed it with a $500 \mathrm{~mm}$ focal length spectrometer equipped with a UV-sensitized charge couple device cooled down with a Peltier module.

\section{Results and Discussion}

High-speed camera images taken at 20 and $38 \mathrm{~ms}$ are shown in Figs. 1(a) and 1(b), respectively. The position of the Macor cap surrounding the $\mathrm{Cu}$ anode is indicated by white dashed lines in Fig. 1(a). The arc current for these two images is about 2 and $0.82 \mathrm{kA}$, respectively. Profiles corresponding to the fourth root of the intensity $(I)$ along the $z=3.5 \mathrm{~mm}$ axis are shown in Fig. 1(c). Note that we plot $I^{1 / 4}$ rather than $I$ as the former quantity can be used to estimate the shape of the arc temperature profile [3]. A decrease in current leads both to a slight decrease in the arc peak emission intensity and to a reduction in the width of the intensity profile along the $y$-axis. As shown in Fig. 1(c), the width of the $I^{1 / 4}$ profile decreases from 9.0 to $7.5 \mathrm{~mm}$ with increasing time from 20 to $38 \mathrm{~ms}$.

Figure 1(d) displays spectra collected at different times from a spot centered in $y=0$ and $z=$ $3.5 \mathrm{~mm}$. With increasing time, one can notice significant changes to the spectra. First, lines at about 505.2 and $506.6 \mathrm{~nm}$ associated with $\mathrm{Cu}$ II states and visible between 20 and $30 \mathrm{~ms}$ cannot be detected for longer times. This decrease in the intensity of ion lines with decreasing current suggests a decrease in the arc temperature. Second, the shape of $\mathrm{Cu} \mathrm{I}$ lines also varies during the experiment. In particular, lines at 515.32 and $521.82 \mathrm{~nm}$ and associated with $\mathrm{Cu} \mathrm{I} 3 d^{10} 4 d^{2} \mathrm{D} 3 / 2$ and $3 d^{10} 4 d^{2} \mathrm{D} 5 / 2$ transitions, respectively, exhibit not only strong variations in their intensity, but also 

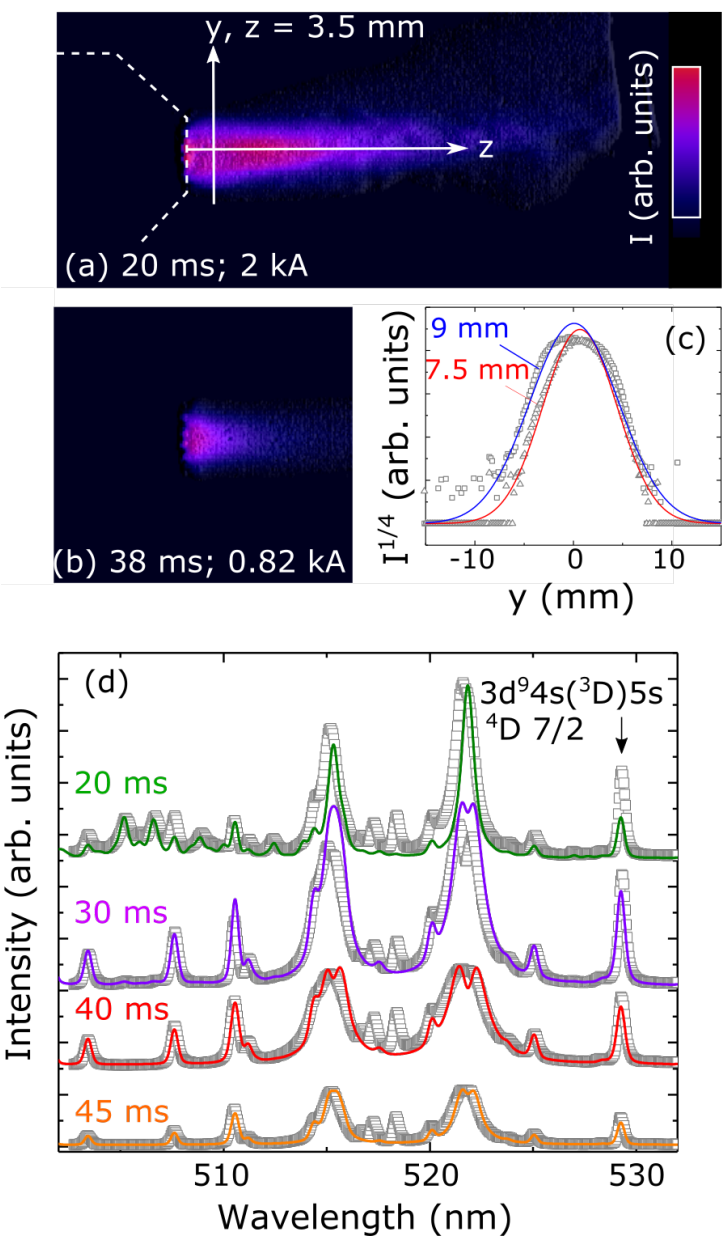
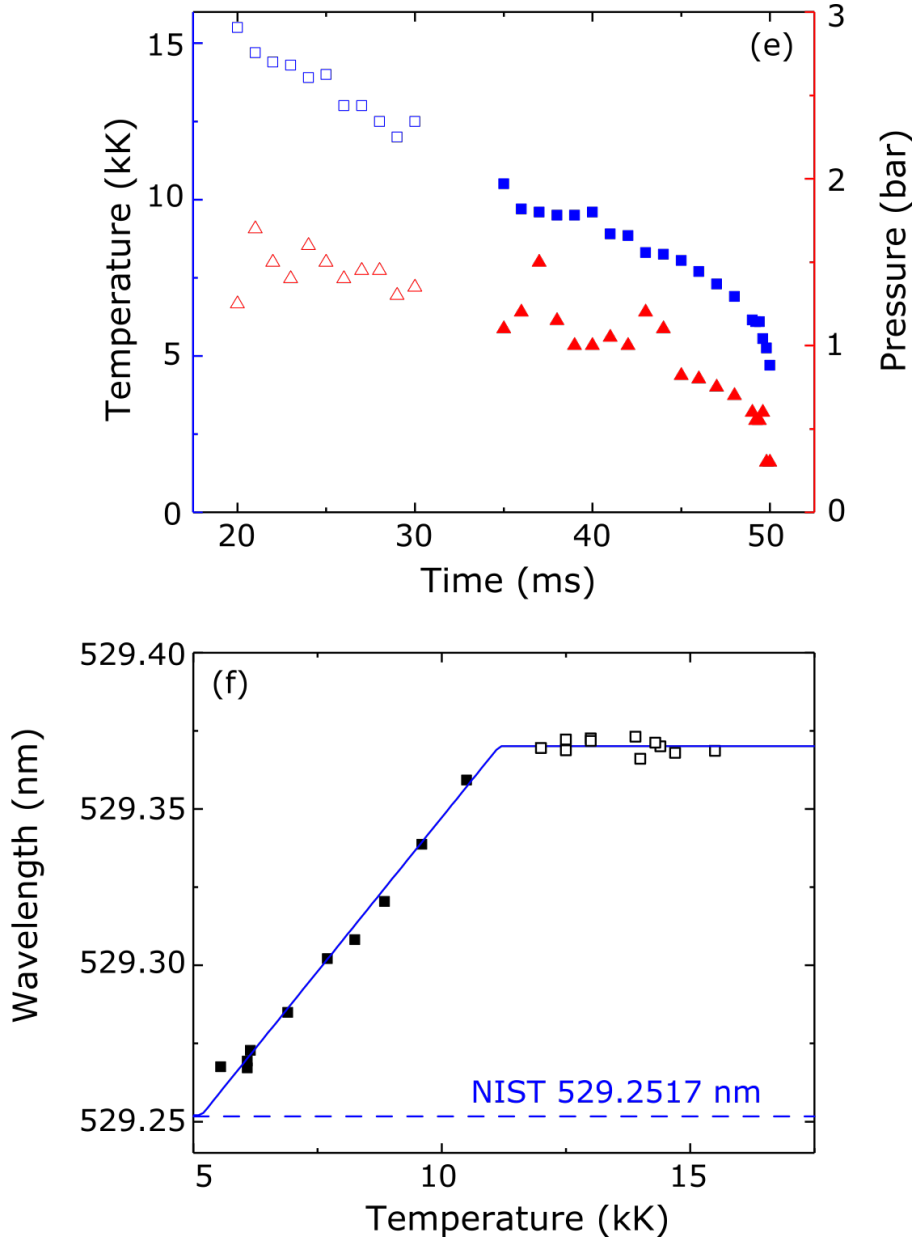

Figure 1. (a,b) High-speed camera images taken at (a) 20 and (b) $38 \mathrm{~ms}$ after ignition and with an arc current of 2 and $0.82 \mathrm{kA}$, respectively. The intensity is color coded according to the linear scale shown in panel (a). The dashed line show the Macor cap surrounding the Cu cylindrical electrode. The $y$ and $z$ axis are also shown. (c) Fourth root of the intensity profile along the $y$-axis at 20 (squares) and $38 \mathrm{~ms}$ (triangles). The blue and red lines are the result of Gaussian fits for the profiles at 20 and $38 \mathrm{~ms}$, respectively. The number on the left show the width of each profile. (d) Spectra detected at 20,30,40 and $45 \mathrm{~ms}$ from the spot at the intersection of the $y$ and $z$ axes in (a). The spectra have been shifted vertically for clarity. The solid lines show the result of the lineshape fit discussed in the text. (e) Time-dependence of the arc peak temperature and pressure. The solid and open symbols are the result of a lineshape fit of the Cu I lines only and of both the Cu II and Cu I lines, respectively. (f) Temperature-dependence of the peak wavelength of the $3 d^{9} 4 s\left({ }^{3} D\right) 5 s{ }^{4} D$ 7/2 line highlighted by an arrow in (d). The dashed line shows the wavelength reported in the NIST database for this transition. The solid lines are guides-to-the-eye.

in their broadening, indicating that both the pressure and the temperature of the arc vary with decreasing arc current.

We described in Ref. [3] a method to extract the time-dependence of the arc peak temperature and pressure from the analysis of high-speed camera images combined with a lineshape fit of the spectra. This method requires several assumptions, that we list in the following: (i) the arc exhibits a radial symmetry, (ii) the width of the fourth-root of the intensity profiles along the $y$-axis in Fig. 1(a) is equal to $0.72 w$, with $w$ the width of the arc temperature profile [3], and (iii) the ratio between $w$ and the width of the arc pressure profiles is equal to 3 [4]. In Ref. [3], we applied this method in the range of temperature where only $\mathrm{Cu} \mathrm{I}$ lines are visible. This range corresponds to times larger than $35 \mathrm{~ms}$ in Fig. 1(c). As shown in Fig. 1(d) for spectra taken at 40 and $45 \mathrm{~ms}$, the lineshape of the detected spectra is well reproduced. The time-dependence of the plasma peak temperature and pressure deduced from the lineshape fit is shown in Fig. 1(e). For times longer than $35 \mathrm{~ms}$, i.e., for arc currents below $1 \mathrm{kA}$, the plasma temperature and pressure at the core of the arc are below $10500 \mathrm{~K}$ and 1.5 bar, respectively.

The method detailed in Refs. [2-4] is not limited to $\mathrm{Cu}$ I plasmas, and we apply it here to times between 20 and $30 \mathrm{~ms}$ when both $\mathrm{Cu} \mathrm{I}$ and $\mathrm{Cu}$ II transitions are detected [(Fig. 1(d))]. The best fits obtained for the 20 and $30 \mathrm{~ms}$ spectra are shown in Fig. 1(d). In view of the assumptions made above, the agreement between simulations and experiments is good. In particular, we reproduce the correct intensity ratio between $\mathrm{Cu}$ II and $\mathrm{Cu}$ I lines. The arc core temperature and pressure 


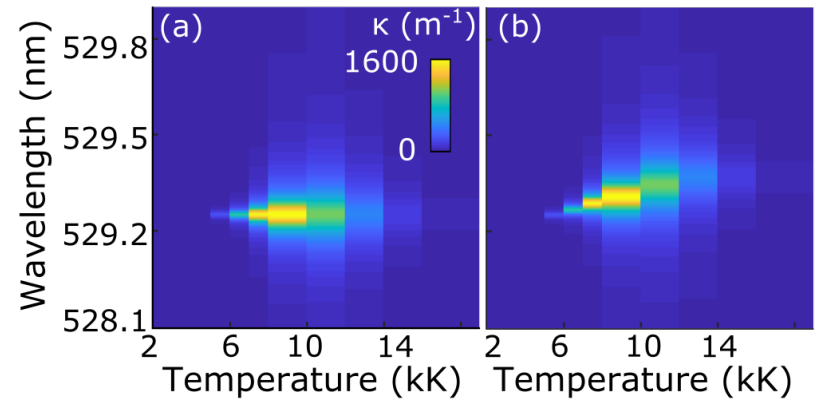

Figure 2. Absorption spectra computed for a 1 bar $C u$ plasma as a function of temperature in the vicinity of the $3 d^{9} 4 s\left({ }^{3} D\right) 5 s^{4} D$ 7/2 line, (a) without and (b) with the temperature-dependent Stark line shift shown in Fig. 1(f). The absorption coefficients $\kappa$ are color-coded according to the linear scale shown in (a).

obtained from the fit of the spectra taken between 20 and $30 \mathrm{~ms}$ are shown in Fig. 1(e). In particular, $20 \mathrm{~ms}$ after ignition and for a current of $2 \mathrm{kA}$, the temperature at the core of the arc exceeds $15000 \mathrm{~K}$.

In addition to the variations in the intensity and broadening of the transitions clearly seen in Fig. 1(d), we could also observe with decreasing current variations in the wavelength of specific lines. Such shifts in the peak wavelength have for instance been reported in Ref. [3] for the $3 d^{9} 4 s\left({ }^{3} \mathrm{D}\right) 5 s{ }^{4} \mathrm{D} 7 / 2$ transition at $529.3 \mathrm{~nm}$. Figure 1 (f) shows the peak wavelength of this line as a function of the arc core temperature shown in Fig. 1(e). On the one hand, for temperatures between 5000 and $10000, \mathrm{~K}$, the peak wavelength of the transition increases linearly with increasing temperature. On the other hand, for temperatures ranging between 12000 and $16000 \mathrm{~K}$, the wavelength of the $3 d^{9} 4 s\left({ }^{3} \mathrm{D}\right) 5 s{ }^{4} \mathrm{D} 7 / 2$ remains essentially constant.

Due to a lack of available data, Stark shifts are usually neglected when computing the absorption spectra of $\mathrm{Cu}$ plasmas. Accordingly, it is not clear how large is the impact of the Stark line shifts reported in Fig. 1(f) on the cooling of the arc by radiation. In particular, one can anticipate the fact that spectral shifts in absorption between the core and the shell of the arc result in reduced reabsorption phenomena and, therefore, in an increased efficiency of the arc cooling by radiation. To estimate how large is the impact of the measured Stark line shifts on the cooling of the arc, we compute in the following the intensity of the $3 d^{9} 4 s\left({ }^{3} \mathrm{D}\right) 5 s{ }^{4} \mathrm{D} 7 / 2$ transition at about $529.3 \mathrm{~nm}$. We perform this calculation with as well as without the temperature-dependent Stark line shift given in Fig. 1(e).

Figure 2(a) shows the temperature dependence of the absorption spectrum computed for a $\mathrm{Cu}$ vapor with a pressure of 1 bar. The Stark line shift was neglected when computing these spectra. In Fig. 2(b), the computed absorption spectra are in addition shifted spectrally according to the results shown in Fig. 1(f). Figure 2(b) thus shows the temperature dependence of the absorption spectrum of
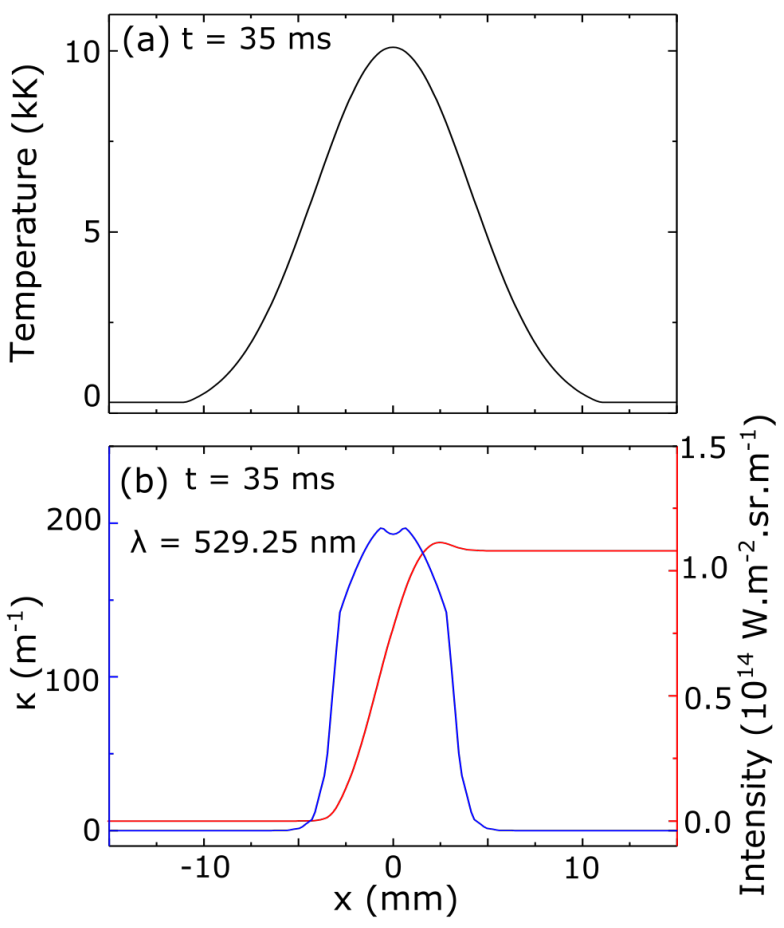

Figure 3. (a) Temperature, (b) absorption coefficient (blue) and intensity at $529.25 \mathrm{~nm}$ (red) profiles for a 1 bar Cu plasma. The profiles have been computed using the peak temperature and pressure shown in Fig. 1(e) for a time $t=35 \mathrm{~ms}$, a temperature profile width $w=$ $8.1 \mathrm{~mm}$ and using the absorption spectra in Fig. 2(a). The intensity profile in (b) has been computed for a ray propagating from negative to positive values of $x$.

a 1 bar $\mathrm{Cu}$ vapor with the Stark line shift taken into account.

Figure 3(a) displays the arc temperature profile along the line of sight using the temperature profile widths and peak values deduced for $t=35 \mathrm{~ms}$ from the high-speed camera images and the spectra. Figure 3(b) shows the evolution of the absorption coefficient of the vapor at $\lambda=829.25 \mathrm{~nm}$, assuming that the vapor exhibits a $\mathrm{Cu}$ fraction of 1 and a pressure of 1 bar. In these simulations, we have used the absorption spectra without Stark line shift shown in Fig. 2(a). In addition, we set $\kappa=0$ for temperatures below the boiling temperature of $\mathrm{Cu}$. With the temperature and absorption profiles in Figs. 3(a) and 3(b), respectively, it is possible using the radiative heat transfer equation to compute the evolution of the intensity of a ray with $\lambda=829.25 \mathrm{~nm}$ that propagates along the lineof-sight. The radiative heat transfer equation reads $\nabla I(x, \lambda)=\kappa(I(x, \lambda)-B(\lambda, T))$, with $B(\lambda, T)$ the Planck function. The one-dimensional profiles in Fig. 3 are discretized and the radiative transfer equation has been solved using simple numerical techniques. The results of the line-of-sight integration is shown in Fig. 3(b). We assume that the intensity obtained in $x=15 \mathrm{~mm}$ in Fig. 3(b) is equal to the intensity of the detected signal $I_{\text {det }}$. Computing $I_{\text {det }}$ for the whole 528 to $530 \mathrm{~nm}$ wavelength range, it is possible to 


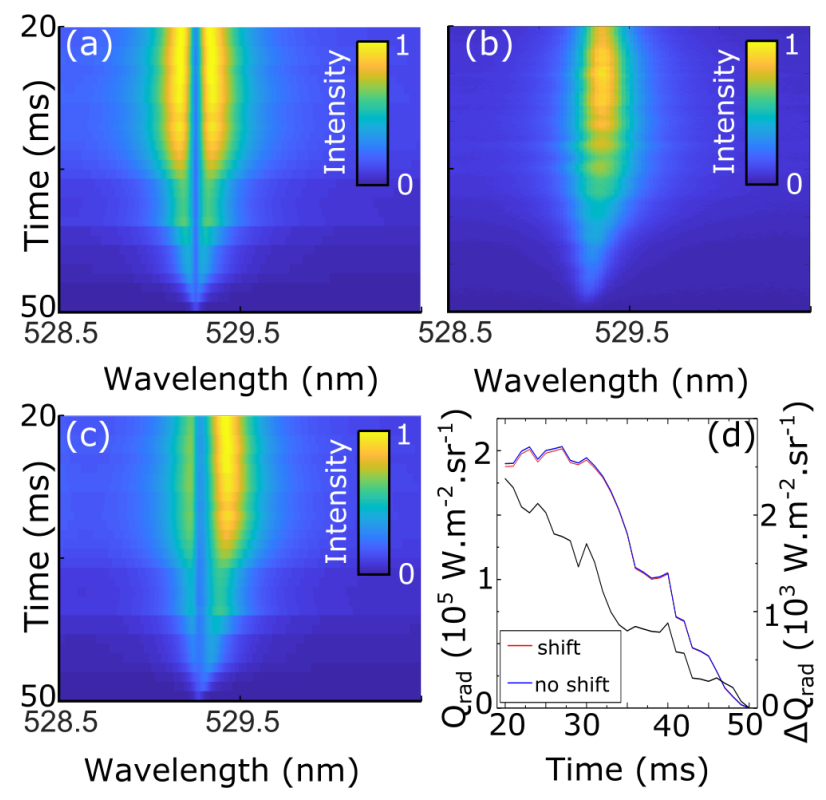

Figure 4. Time-dependence of the spectra (a) computed for a 1 bar Cu plasma, neglecting the temperaturedependent Stark line shifts in Fig. 1(f). The intensities are color-coded according to the linear scale on the right. The measured time-dependent spectra are shown in (b). (c) Same as (a), this time accounting for the temperature-dependent Stark line shift in Fig. 1(f). (d) Radiation intensity $Q_{\text {rad }}$ spectrally integrated between 528.5 and $530.5 \mathrm{~nm}$ in $x=15 \mathrm{~mm}$ without (red) and accounting for (blue) the Stark line shift of the $3 d^{9} 4 s\left({ }^{3} D\right) 5 s{ }^{4} D 7 / 2$ transition. The intensity difference $\Delta Q_{\text {rad }}$ between the cases with and without Stark line shift is shown by the black line.

deduce the exact lineshape of the $3 d^{9} 4 s\left({ }^{3} \mathrm{D}\right) 5 s{ }^{4} \mathrm{D} 7 / 2$ transition. The result of our calculations are shown in Fig. 4(a).

In agreement with the experimental observations shown in Fig. 4(b), the line intensity remains essentially constant between 20 and $35 \mathrm{~ms}$, and decreases for longer time. The constant intensity of the detected signal for times between 20 and $35 \mathrm{~ms}$ is related to the combination of the decrease in temperature from 15500 to $10500 \mathrm{~K}$ and the increase in peak absorption coefficient from 40 to above $10^{3} \mathrm{~m}^{-1}$. For times longer than $35 \mathrm{~ms}$, the detected line not only weakens, but also narrows. The latter observation arises from a reduction in Stark broadening that result from the decrease in pressure displayed in Fig. 1(e). Note that the pronounced absorption dip in the spectra in Fig. 4(a) is smeared out by the response of our spectrometer and is therefore not observed experimentally. Also, while there is a clear blueshift in the peak wavelength of the measured $3 d^{9} 4 s\left({ }^{3} \mathrm{D}\right) 5 s^{4} \mathrm{D} 7 / 2$ line with increasing time [Fig. 4(b)], the central wavelength of the computed transition in Fig. 4(a) remains constant.

We apply the procedure described above to compute the time-dependence of the lineshape of the $3 d^{9} 4 s\left({ }^{3} \mathrm{D}\right) 5 s{ }^{4} \mathrm{D} 7 / 2$ transition using the shifted absorption spectra in Fig. 2(b). As shown in Fig. 4(c), we reproduce the experimental observation of a constant peak wavelength for times below $35 \mathrm{~ms}$, together with the blueshift of the transition for longer times. As for the spectra without Stark line shift, a pronounced reabsorption dip is observed in the simulated spectra shown in Fig. 4(d). We emphasize, however, that in contrast to the peak wavelength, the spectral position of this dip remains constant between 20 and $50 \mathrm{~ms}$. Consequently, it is mostly related to light reabsorption occurring in the cold shell of the arc.

To quantify the impact of the observed Stark line shifts on the heat transfer by radiation, we integrate spectrally the spectra in Figs. 4(a) and 4(c). The time-dependence of the spectrally integrated intensity obtained this way is plotted in Fig. 4(d). For all times, the intensity of the detected signal is the strongest when accounting for the Stark line shift. This finding is in agreement with the expected increase in cooling by radiation in the presence of Stark line shifts. This intensity increase is, however, rather small. As shown in Fig. 4(d), for times between 20 and $35 \mathrm{~ms}$, i.e., when the impact of the Stark line shift is the largest [Fig. 4], the intensity increase is below $2 \%$.

\section{Conclusions}

In conclusions, the wavelength of $\mathrm{Cu}$ I $3 d^{9} 4 s 5 s$ transitions in $\mathrm{Cu}$-dominated plasmas increases nearly linearly between 5000 and $10000 \mathrm{~K}$ as a result of the Stark effect and remains essentially constant at higher temperatures. The spectral detuning in absorption between the hot core and cold shell of the arc results in a reduction in radiation cooling of the plasma. We have shown that this reduction is, however, below $2 \%$. The Stark shift of $\mathrm{Cu}$ transitions can thus be neglected when simulating the dynamics of arcs in low-voltage switching applications.

\section{References}

[1] H. Nordborg and A. A. Iordanidis. Self-consistent radiation based modelling of electric arcs: I. efficient radiation approximations. Journal of Physics D: Applied Physics, 41(13):135205, 2008. doi:10.1088/0022-3727/41/13/135205.

[2] S. Franke, R. Methling, D. Uhrlandt, R. Bianchetti, R. Gati, and M. Schwinne. Temperature determination in copper-dominated free-burning arcs. Journal of Physics D: Applied Physics, 47(1):015202, 2013. doi:10.1088/0022-3727/47/1/015202.

[3] P. Corfdir, G. Lantz, M. Abplanalp, T. Delachaux, F. Kassubek, P. SÃijtterlin, and M. Bator. Stark shift measurement as a temperature diagnostic of cu-dominated thermal plasmas. Journal of Physics D: Applied Physics, in press, 2019. doi:10.1088/1361-6463/ab188e.

[4] M. Abplanalp, K. Menzel, T. Delachaux, R.-P. SÃijtterlin, and F. Kassubek. Optical investigation of constricted vacuum arcs. In 27th International Symposium on Discharges and Electrical Insulation in Vacuum (ISDEIV), volume 1, pages 1-4, 2016. doi:10.1109/DEIV.2016.7748731. 